would then also have an opportunity to understand the pathologic changes in the mouth coincident with pregnancy. There is a way, she said, to educate people to appreciate the importance of the care of their teeth. The way to educate the mothers is to begin at the mothers' meetings and teach them the whys and wherefores of these things. They must be shown that these reflex pains and the constant nagging and irritation is hurting the nervous system of the mother as well as that of the child.

Dr. Wilber M. Dailey, New York City, inquired whether any careful observation has been made of the mouth previous to pregnancy. He has noticed in many cases where the mouth has been well cared for, and looked after in a scientific and prophylactic manner, that there has been little or no distintegration of tooth structure during pregnancy. Of course, he said, where there is caries and considerable calcareous deposit present, the teeth are more or less affected. Dr. Dailey questioned the correctness of the statement that there is a certain amount of breaking down of lime salts in different parts of the body, and a rebuilding, a chemical and pathologic change. He pointed out, in the nature of prophylaxis, that if physicians would examine the secretions as well as the excretions of their patients, annually or semi-annually, or even monthly, any pathologic change could be discovered and treated. And, likewise, if this practice were followed with relation to conditions in the buccal cavity, the exact changes taking place during pregnancy could be determined.

Dr. S. B. Luckie, Chester, Pa., spoke of the value of such papers as that of Dr. Power in affording physicians an opportunity to become familiar with what dentists think of such eases. He agreed with what the essayist said in regard to the treatment of the mouth during pregnancy. He has never hesitated to perform any necessary dental operation during that period, proviled he had the full confidence of the patient. The extraction of teeth or the treatment of pulpitis can be undertaken. The treatment of pulpitis and the percementation of the teeth can very often be attended to in a temporary way for a few months until the time arrives when permanent work can be done. Pulpitis can often be controlled for several months by the use of iodoform or formaldehyd or thymol and, possibly, indin. These agents can be sealed in the cavity and absorbed by the dentine, setting up an antiseptic condition that will often keep the tooth comfortable for some time.

Dr. JAmes E. Power, Providence, R. I., said that the principal reason for presenting his paper was the fact that it may be read by physicians, and in that way reach hundreds of thousands of people whom dentists would otherwise be unable to reach. The mental condition of the pregnant woman should receive a greater consideration. It should be viewed just as carefully as are the physiologic conditions which are superinduced by pregnancy. In this way the confidence of the patient is secured and treatment will be much more effective. Dr Power stated that he always found the acidity more marked where there was a profuse salivation, but added that he took more tests in cases of profuse salivation than where the salivation was apparently normal. He confines himself to the use of local anesthesia almost entirelv. There is, he said, always a variation in the manner in which gas is absorbed by different people. In pregnancy there is danger of producing convulsions and abortion. By using the local anesthetic this danger is eliminated, and all other dangers are reduced to a minimum. The first step in the injection of a local anesthetic is to wash the surface through which the needle is to be introduced, in the same manner as one would treat the surface of the abdomen if he were going to perform a laparotomy. The syringe is sterilized and filled, and the needle is inserted into the gum tissue. Ry withdrawing the needle just a little a space is made between the end of the needle and the tissue; then the anesthetic is forced gradually into the tissue, the needle being reintroduced within the whitened area. The tooth should not be extracted until anesthesia is complete, so as not to produce mental shock. Dr. Power emphasized the value of the frequent ingestion of small amounts of food, because a greater amount of food can be taken in a given time if divided in small doses than can be taken in a few large meals. $\mathrm{Dr}$. Power has observed that the previous eare that has been taken of the patient's teeth makes little difference in the condition of the teeth in the pregnant woman. He believes that the physiologic process changes to a pathologic one, regardless of the previous condition or habits of the individual as far as the teeth are concerned.

\section{PIIYSICAL DIAGNOSIS: URGENT NEED OF REVISION.}

\section{J. H. TYNDALE, M.D. LINCOLN, NEB.}

The collective title of "physical diagnosis" is applied in its narrowest sense to ascertaining the condition of the lungs, heart and large blood vessels-the contents of the thoracic cavity.

For the past two decades simplification of methods employed in physical diagnosis has been advocated by leading specialists in pulmonary diseases, Dr. Charles E. Quimby of New York City, being the foremost champion of the reform.

It is a common complaint on the part of advanced students that old legends which were taught as truths a generation or more ago still survive in the text-books and are still accepted by a great majority of the profession. It ought not be necessary to reconcile ourselves to the idea that still another generation will pass before the truth will filter down from specialists in lung diseases into the text-books.

He is a benefactor to the people who eradicates weeds from the farm and errors from the mind-in this instance the medical mind. By this token, if those of us who stand for revision can demonstrate to and convince the profession that we not only simplify methods and cut off more than two-thirds of useless terms, but arrive at a more sharply defined diagnosis than by the old methods, it stands to reason that revision is urgently needed.

'To the insistent and somewhat inconsistent therapeutical cry of "back to nature" we insist on the amendment "back to physical diagnosis." And why? For the following sound and solid reasons :

1. In lung destruction the tubercle bacillus is a guest invited by the undernutrition of the host and marking an advanced stage of disease.

2. The tuberculin reaction is, at the present day, scarcely considered available for the diagnosis of human tuberculosis.

3. Thus far the Roentgen-rays have added nothing of any special value to older methods of physical exploration, save under the heading of "confirmatory."

4. Râles, rhonchi, with their countless adjectives pointing to their composition and locality, should be relegated to the background as concomitant features, not an underlying condition.

In the main, then, diagnosis of tuberculous invasion of the lung is as much dependent on skill in auscultation as it has ever been.

How early can we recognize the first beginnings of tuberculous invasion of the pulmonary apparatus? Can any additional signs be elicited by auscultation to fix the locality of the tuberculous invasion indicated by slowly progressive anemia or emaciation or persistent lassitude? The answer to the first question is: Just as early as the patient complains of anything, even if it be only getting tired easily. To the second: Quite as early as in advanced cases.

A verdict in diagnosis will not stand unless it is based on the combined values of general and local features. 
In order of importance the general features rank as follows:

1. Persistent lassitude, attributed by the patient to his legs, but in truth based on accelerated tempo of the heart's action, with or without excess of central propulsion over arterial tension.

2. Progressive and persistent anemia, unaccounted for by other invasions of infection, inflammatory processes, central or peripheral nervous disturbances or, last but not least, stubborn stomachic or intestinal indigestion. This latter element should always be removed before a diagnosis is ventured on.

3. Progressive and persistent emaciation demands the same cxclusions as recorded above. Frequent weighings alone are to be relied on, not the opinion of the patient nor that of his friends. The accepted general division of tuberculous and other forms of pulmonary consumption is into "open" and "closed" cases. An open case is one in which the products are open to and communicate with the atmosphere. Here evacuation and not "walling in" is imperatively indicated, as will be shown presently.

A closed case is one not accessible to the atmospheric air. Tuberculous pleurisy and enlarged glands may serve as examples.

For prognostic and therapeutic purposes, both open and closed cases will be found in the organism to exist as two very distinct and separate pathologic conditions, namely:

(a) Conditions inherent in the lymphatic channels. As a distributing center of tuberculous infection the bunches of tracheobronchial glands rank first. Richest in lymph vessels of all serous membranes is the pleura - "the dumping ground of the lymphatic system."

(b) Tuberculous or streptococcic infection engrafted on unresolved remnants of previous acute diseasecroupous and catarrhal pneumonia, pleurisy, ete.

The products of both pathologic conditions are pus or connective tissue or both.

The difference between ancient methods and the modern scheme of simplification is best shown by placing them in sharp contrast. But I find that enumeration of old terms would fill a moderately sized monthly magazine (take the twenty kinds of râles as an instance) and hence proceed to present the modern method in the order practiced in making a diagnosis.

\section{auscultation.}

Auscultation recognizes only "tonality" and "pitch" as representing the sounds (or melody, if you prefer) heard in inspiration and expiration. The mechanical movements - the technical execution - are represented by "rhythm" and "tempo."

Tonality or the Keynotes.-Of these there are only two in the lung-pulmonary and bronchial, or, in other words, vesicular and tubular. These are the fundamental tones.

Pitch.-This differs from musical pitch by being relative. Trachegl breathing is our standard, as being the only fixed standard-inspiration and expiration as nearly as possible of the same pitch. In auscultation it represents the highest type of pitch, and all others are gauged by it as being "lowered" or "raised" in the locality heard. Three degrees of either are sufficient for correct diagnosis, as, for example: Pitch lowered in first, second or third degree. Pitch is never an adjunct of vesicular or tubular breathing. Brought about by the sum total of respiration, it is an entirely independent factor.
Rhythm.--Rhythm is the regular recurrence of accents, the characteristic of regular succession. It is punctuation. The rhythm of breathing in the normal condition of the lung is a regular succession of marked accents. It consists, as we all know, of inspiration, pause and expiration-what in music would be called a three-part rhythm. The rhythm of respiration is a continuous movement (legato), while that of the heart is interrupted (staccato). A change from the continued to an interrupted rhythm marks one of the earliest beginnings of a pathologic condition in the lung, both in infections and inflammations.

Tempo.-Tempo is nothing more or less than the speed of the rhythm. The normal tempo of breathing is from sixtcen to eighteen respirations to the minuteabout one to every four seconds.

\section{RALES.}

The importance attached to râles or rhonchi is another sacred tradition. Transudations and secretions accompanying functional or organic disease of the lungwhether serum, mucus, pus or blood- are not the pathologic condition itself, nor do they give us any definite clue to the same. Their expectoration or absorption still leaves the condition to be ascertained by tone, pitch, rhythm and tempo - four designations, as against twenty-seven of the old nomenclature.

\section{PERCCSSION.}

Percussion recognizes resonance only. Four degrees of resonance-excessive resonance or reduced (deficient) resonance-are ample to describe existing conditions. There is no such thing as pitch in percuseion, other than applies to the chest walls.

\section{INSPECTION.}

Inspect for nothing but the anatomic sufficiency of the thorax, which sufficiency should bear some relation to the height of the patient, but not very much. Judge only by an abnormally narrow thorax or an equally abnormally wide one. The next step suggests itself. By a few deep inspirations (never mind the tape line and the number of inches) ascertain the physiologic sufficiency. Remember these facts: A narrow thorax may be capable of unlooked-for expansion, while a large barrel-shaped one may scarcely move at all-the socalled fixed thorax. What we are concerned with is the elasticity of the costal cartilages.

\section{PALPATION.}

Palpation is only useful for ascertaining the amplitude of the diaphragmatic excursions-the phrenic wave so-called. Remember that lateral, not anteroposterior expansion, is the gauge of sufficient respiration.

\section{MAXIMS TO BE BORNE IN MIND.}

Five general maxims are to be kept in mind. They are:

1. Raised pitch in auscultation, coupled to reduced resonance, denotes condensation.

2. Lowered pitch, coupled to exaggerated resonance, denotes expansion.

3. Both condensation and expansion of lung tissue lead up to the loss of elasticity. Temporary loss is a phase of healing processes. Permanent loss is one of the terminal phases of disease.

4. The degree of percussion resonance establishes the exact value of the respiratory pitch obtained by auscul- 
tation. Percussicin either confirms or negatives the auscultation find-the keynote of differential diagnosis between active and ended processes.

5. Areas or foci of infection or inflammation or both, demonstrated by auscultation and confirmed or not by percussion, exist either beneath the locality examined or are the result of mechanical hindrance to respiration.

How many practitioners ever discover and take cognizance of diaphragmatic pleural adhesions? Is it not true that diminished respiration in the upper lobes of the lung is invariably placed to the credit of an infiltration when, as a matter of fact, in the great majority of those cases full respiration is made impossible by traction of pleuritic adhesions in the axillary region?

Pleuritic adhesions have two attributes. They are permanent and progressive. Thickenings already on hand are there to stay and gradual encroachment from their periphery to uninvolved tissue is the rule.

For convenience sake, let me speak of pleuritic adhesions as linear and flat. The former occur along the inner edges of the sternum, the inner edge of the scapula and, last but of the utmost importance, on the upper surface of the diaphragm. The flat variety occurs in the infra-axillary region and is the most aggressive as to upward progression.

I am well within the truth in saying that the most neglected field in medicine and the one in which most slipshod diagnoses are made comprises the existence, effects and treatment of pleuritic adhesions and their mechanical effects. Of equal importance as a mechanical hindrance is the "albuminous agglutination" (Dr. Abrams, San Francisco) of the peripheral pleural surface to the chest walls with its accompanying anemia from continued traction and possible gangrene of parts involved -a frequent phase of consumption.

"Nature's barriers" is the term applied to the above processes. as well as the encapsulation of tuberculous foci. Adhesions may be a defense, but not a curative defense. On the contrary, they are part and parcel of the invasion-extension of the inflammatory process, inseparable from tuberculous infection. In other words, adhesions, tuberculous or rheumatic, are not constructive but destructive and call for removal wherever found. Liberating the imprisoned lung (by hypodermic injection of sodium cinnamate) should always precede the now firmly established vaccine therapy by immunization with watery extract of tubercle bacilli.

Modern methods seek to inculcate that from the earliest beginnings of pulmonary consumption to the most obvious and readily ascertainable manifestations this is the sequence:

1. Permanently accelerated tempo of the heart's action, not due to peripheral resistance in the liver or kitineys or demonstrable in the lung, associated with one, two or all three of the general features, lassitude, anemia, emaciation; temperature or no temperature; microbes or no microbes. This is the true skirmish line of pulmonary consumption. Presence or absence of high temperature and of tubercle bacilli, even when sputum is abundant, likewise holds good in the more advanced forms of pulmonary consumption. I note this to avoid repctition.

2. Permanent change of rhythm associated with lassitude, anemia or cmaciation. singly or combined. The ancient method names these changes: Cogwheel respiration, prolonged pause, audible and prolonged expiration. Modern dictum: Inspiration, nause, and expiration may singly or in unison be involved. The path- ologic changes of rhythm are simply the reverse of the physiologic, i. e., smoothly flowing audible inspiration converted into an interrupted (staccato) one, a prolonged pause where previously inspiration glided directly over into expiration, now displaced by a prolonged and audible one. Cogwheel respiration and cracked-pot sound are illustrations of the truism: "Comparisons are odious."

3. Changes in pitch, heard permanently over a given area. At this stage of disease development a diagnosis is complete even without the general features of lassitucle, anemia or emaciation. On the other hand, percussion must confirm the find by deficient resonance (the old impaired resonance or dullness). Normal or excessive resonance denotes an emphysematous (dilated) patch overlying a healed or healing area.

Note this law: Pitch is always "raised" in progressive disease until it merges into a change of tonality (tubular breathing). This pitch raise is always relat ve -namely, gauged by the tracheal pitch. Pitch in health is "lowered" from the standard and "raised" in disease.

Diagnosis by pitch relation includes all processes in the pleura, be the contents solid, liquid or air. The folly of percussing first and auscultating afterward is markedly illustrated by contents of the pleura. In either case we have the highest degree of pitch (silence). requiring a differential diagnosis by pereussion. Hxcessive resonance for air, dulness for solid contents, flatness for fluid. Percussion first is putting the cart before the horse.

4. Permanent change of tonality from the pulmonary to the bronchial, from diffused to concentrated tubular breathing. Complete solidification or excavation (cavity), decp seated or near the surface; single or multiple; or advanced shrinkage, overlaid or not by an emphysematous patch, call for no particularly fine adjustment in auscultation. The changes in tonality-the "quality" of ancient writings-can be heard by any one with an untrained ear. Incidentally, it is always woll to remember that bronchial or tubular breathing is present not only in pathologic but in physiolngic conditions as well. In the latter it is drowned by the low-pitched pulmonary breathing. As with pitch, a diagnosis is here complete in the absence of general features.

There is no need of such terms as "cavernous" or "amphoric" breathing or of the terms named above.

Note the presence of râles only as an incident and as indicative of furnishing sputum. Drop the terms sibilant, sonorous, gurgling, bubbling. coarse, mucous, fine. crepitant or subcrepitant. Every instructor in physical diagnosis knows full well that this multiplicity of terms only confuses and disheartens the students. And what is more to the purpose, these terms are of no earthly value in arriving at a clean cut diagnosis.

"Let the dead past bury its dead."

The Bad Habit of Using Purgatives.-The Indiana Medical Journal agrees with Dr. Edwin Walker of Evansville, who teaches that purgatives are responsible for most of the constipation of the people who use them. They have the pill-purgative propensity, and are educated to the practice and fortified in their use by the physician. The present purgative habit of American people is the natural outcome of intemperance in diet, of the use of concentrated food and ton much of it of physical laziness and mental apathy. It is far better to teach people to abandon purgatives than to use new ones. Modern pharmacy has done much for medicine, but too much in the way of purgatives. 